\title{
氧化铝富集糖肽的研究
}

\author{
赵旭 $^{a}$ 姜武辉 ${ }^{c}$ 于龙 $^{b}$ 邹丽娟*, $a$ 李秀玲 ${ }^{*}, b$ 梁金金沝 $b$ \\ $\left({ }^{a}\right.$ 大连医科大学附属第二医院 大连 116023) \\ $\left({ }^{b}\right.$ 中国科学院大连化学物理研究所 中国科学院分离分析化学重点实验室 大连 116023) \\ (c辽宁宇洁环保咨询有限公司 沈阳 110000)
}

\begin{abstract}
摘要 糖基化蛋白对于生命体的生长发育, 免疫调节, 细胞识别粘附等具有重要意义, 而异常的糖基化表达与风湿关 节炎、肿瘤、阻塞性肺病等疾病密切相关. 因此糖蛋白结构检测对于研究生命活动至关重要. 由于在复杂样品中糖肽 含量相对较少，加之非糖肽的离子抑制作用，使得糖肽的质谱检测有一定的挑战性. 因此发展一种有效富集糖肽的方 法是必要的. 本实验中我们选用氧化铝对糖肽进行富集研究, 并考察了影响氧化铝保留多肽的机理. 我们利用氧化铝, 从 $\mathrm{HRP}$ 酶解液中共获得 16 个糖肽, 从 $\mathrm{IgG}$ 酶解液中共获得 12 个糖肽. 与直接检测样品酶解液和经商品化材料 Sepharose 富集后再检测相比, 检测到糖肽的个数增多. 实验数据证明氧化铝富集糖肽具有较好的选择性和覆盖率. 关键词＼cjkstart氧化铝；糖肽；富集；质谱; 多肽
\end{abstract}

\section{Selective Enrichment of Glycopeptides Using Aluminum Oxide}

\author{
${\text { Zhao, } \mathrm{Xu}^{a} \quad \text { Jiang, Wuhui }^{c} \quad \text { Yu, Long }^{b} \quad \text { Zou, Lijuan*,a }^{*} \quad \text { Li, Xiuling }}^{*, b} \quad$ Liang, Xinmiao $^{b}$ \\ $\left({ }^{a}\right.$ The Second Hospital Affiliated to Dalian Medical University, Dalian 116023) \\ $\left({ }^{b}\right.$ Key Lab of Separation Science for Analytical Chemistry, Dalian Institute of Chemical Physics, \\ Chinese Academy of Sciences, Dalian 116023) \\ ( ${ }^{c}$ Liaoning Yu Jie Environmental Consulting Limited Company, Shenyang 110000)
}

\begin{abstract}
Protein glycosylation is significantly associated with cells cycle, immune regulation, cells recognition and cells adhesion. Aberrant glycosylation expression is involved in many diseases, such as rheumatoid arthritis, cancer, and chronic obstructive pulmonary disease. Thus, characterization of the protein glycosylation is very important to understand the life process. However, detection of glycopeptides is difficult by mass spectrometry because of the low concentration in complex sample and the suppressed signal by non-glycopeptides. Therefore, it is essential to explore an effective technique for glycopeptide enrichment. In this study, an alumina based-materials was used to selectively enrich glycopeptides. Firstly, we investigated the retention mechanism by changing the concentration of acetonitrile (ACN). With the decreased concentration of $\mathrm{ACN}$, it was found that peptides were eluted according to their hydrophilicity. Moreover, most of the non-glycopeptides were eluted earlier than the glycopeptides in the high concentration of ACN fraction and the glycopeptides were found in the low concentration of ACN fraction. This result proved that hydrophilic interaction is one of the retention mechanisms for peptides retained by alumina. Secondly, we investigated the retention mechanism by changing the concentration of ammonium hydroxide. ACN were fixed at a high concentration and peptides were subsequently eluted with different concentration of ammonium hydroxide solution. At a low concentration of ammonium hydroxide solution, the peptides were no found. But at a high concentration of ammonium hydroxide solution, the glycopeptides and non-glycopeptides were eluted simultaneously. This proved that the ligand exchange is also one of the retention mechanisms, where the retention of the glycopeptides and non-glycopeptides showed no different under this mechanism. Based on the above mentioned results, the enrichment condition was optimized under the model of solid-phase extraction. High concentration of ACN mixed with a certain concentration of ammonium hydroxide solution as loading buffer and low concentration of ACN mixed with a certain concentration of ammonium hydroxide solution as elution buffer. The number of glycopeptides enriched by alumina were compared with glycopeptides before enrichment and enriched by Sepharose. In the tryptic HRP digest, 7 glycopeptides were found before enrichment, 16 glycopeptides were found by alumina and 14 glycopeptides were found by Sepharose. In the tryptic IgG digest, 2 glycopeptides were found before enrichment, 12 glycopeptides were found by alumina and 4 glycopeptides were found by Sepharose. In conclusion, alumina based-method used to enrich glycopeptides has high selection and wide coverage.

Keywords alumina; glycopeptides; enrichment; mass spectrometry; peptides
\end{abstract}

*E-mail: zoulijuan1963@sina.com; lixiuling@dicp.ac.cn

Received December 26, 2012; published January 25, 2013.

Project supported by the National High Technology Research and Development Program 863 (No. 2012AA020203), the National Natural Science Foundation of China (Nos. 81171486, 21105100) and State Key Laboratory of Advanced Materials Synthesis Processing (Wuhan University of Technology, 2013-KF-8). 项目受国家高技术研究发展计划 863 计划(No. 2012AA020203)、国家自然科学基金(Nos. 81171486, 21105100)和材料复合新技术国家重点实验室(武 汉理工大学, 2013-KF-8)开放基金资助. 


\section{1 引言}

糖基化是蛋白质翻译后修饰的一种重要形式, 并参 与生命体的生长发育、免疫调节、细胞识别粘附等过程 ${ }^{[1]}$. 异常的糖基化表达与许多疾病相关, 如: 风湿关节炎 ${ }^{[2]}$, 肿瘤 ${ }^{[3]}$, 阻塞性肺病 ${ }^{[4]}$ 等. 因此, 检测蛋白质的糖基化位 点对于研究生命活动非常重要 ${ }^{[5]}$. 质谱因其高通量和高 灵敏度成为检测糖基化多肽(糖肽)的重要手段 ${ }^{[6]}$. 但是 由于糖肽本身含量较少且离子化效率低, 加之非糖肽对 其有离子抑制效应，使得其在质谱上的检测灵敏度低， 难于检测 ${ }^{[7]}$. 因此, 发展一种有效富集糖肽的方法是极 其必要的. 肼化学法富集糖肽特异性高, 但会造成糖链 信息丢失, 而用硼酸富集则会结合非糖肽, 导致质谱信 号的干扰 ${ }^{[8]}$. 亲水作用色谱法富集糖肽的覆盖面较高, 但特异性有所不足. 凝集素应用最广, 特异性强, 但只 对某类糖型起作用 ${ }^{[9]}$. 由于上述方法各有不足, 因此需 要发展一种新的方法来提高糖肽的选择性和覆盖率.

金属氧化物具有独特的表面化学性质和稳定的机 械强度. 包括氧化钛、氧化锆和氧化铝在内的金属氧化 物都已被用于正相色谱中分离极性化合物 ${ }^{[10]}$. 不仅如 此, 氧化钛和氧化锆还被用来选择性富集糖肰 ${ }^{[11,12]}$. 氧 化铝与其他金属氧化物相比具有相似的理化性能, 但一 直未被用于对糖肽的富集. 所以, 本文以氧化铝为材料 对其富集糖肽进行研究. 首先考察亲水作用和配基交换 作用对多肽保留的影响. 在此基础上, 优化氧化铝富集 糖肽的方法.

\section{2 结果与讨论}

\section{1 考察亲水作用对多肽保留是否有影响}

本实验以西格玛公司酸性氧化铝(规格: 150 目)为 富集材料, 以经胰蛋白酶酶解 ${ }^{[11]}$ 后的辣根过氧化物酶 (HRP)为样品, 考察乙腈(ACN)浓度变化对多肽保留影 响. 取 $2 \mathrm{mg}$ 氧化铝材料填装到凝胶吸头中, 制成微型固 相萃取柱(SPE). 然后将 $20 \mu \mathrm{L}$ HRP 酶解液旋干, 再溶于 $80 \% \mathrm{ACN}$ 后上样, 样品采用 $80 \%, 70 \%, 60 \%, 50 \%$ 乙腈依 次洗脱. 各洗脱馏分经四级杆-飞行时间质谱检测, 所 得质谱图如图 1 所示.

从图 1 可以看出随着乙腈浓度的降低, 溶剂的洗脱 能力逐渐增加. 在 $80 \%$ 乙腈馏分中没有多肽被洗脱出, 而在 $70 \%$ 乙腈到 $50 \%$ 乙腈馏分中多肽逐渐被洗脱出. 另 外, 在 $70 \%$ 乙腈馏分中洗脱下来全部都是非糖肽. 在 $60 \%$ 乙腈馏分中可以看到 2 个糖肽，而在 $50 \%$ 乙腈馏分 中除了上述 2 个糖肽外还发现另外 2 个糖肽. 这表明, 随着乙腈浓度的降低, 疏水性肽和极性较强的糖肽先后 被洗脱下来，说明氧化铝对多肽具有亲水保留作用.

\section{2 考察配基交换作用对多肽保留是否有影响}

氧化铝具有路易斯酸性, 而多肽在碱性环境中显路 易斯碱性，用氨水这种更强的路易斯碱可以洗脱多肽.
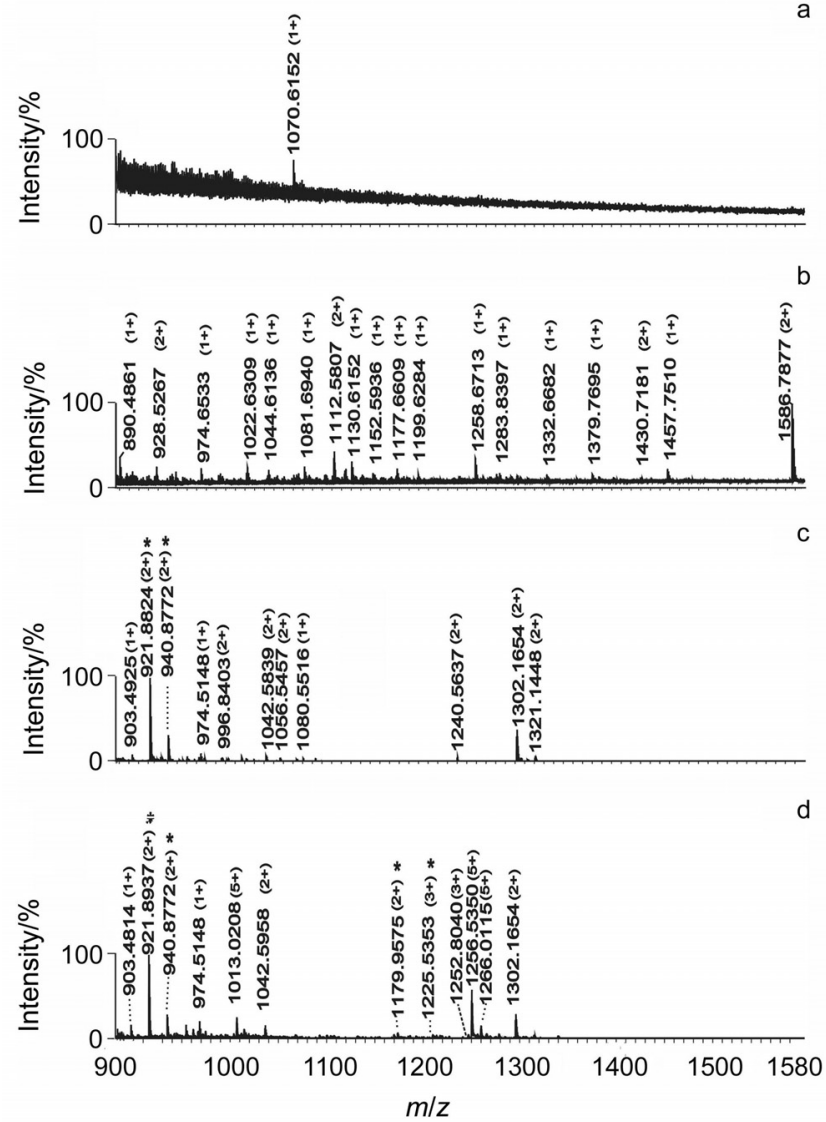

图 $1 \mathrm{HRP}$ 酶解液上样到氧化铝微柱后, 经 (a) $80 \% \mathrm{ACN}$, (b) $70 \% \mathrm{ACN}$, (c) $60 \% \mathrm{ACN}$ 和(d) $50 \% \mathrm{ACN}$ 顺序洗脱后的质谱图. “*” 为 糖肽，非糖肽以质荷比 $(m / z)$ 表示

Figure 1 Mass spectra of tryptic digest of HRP loaded into $\mathrm{Al}_{2} \mathrm{O}_{3}$ and subsequently eluted with (a) $80 \% \mathrm{ACN}$, (b) $70 \% \mathrm{ACN}$, (c) $60 \% \mathrm{ACN}$ and (d) $50 \% \mathrm{ACN}$. Glycopeptides are marked with "*”. Other peptides are marked with their $m / z$ values

利用这个原理, 我们同样以 HRP 酶解液为样品, 固定 $80 \%$ 乙腈浓度后改变氨水浓度为 $1 \%, 2 \%, 3 \%$ 顺序洗脱. 各洗脱馏分经四级杆-飞行时间质谱检测所得质谱图如 图 2 所示. 由于在亲水模式 $80 \%$ 乙腈条件下多肽没有被 洗脱下来, 所以我们把乙腈的浓度固定到 $80 \%$.

从图 2 可以看出在 $80 \% \mathrm{ACN} / 1 \% \mathrm{NH}_{3} \cdot \mathrm{H}_{2} \mathrm{O}$ 条件下没 有肽被洗脱下来，在 $80 \% \mathrm{ACN} / 2 \% \mathrm{NH}_{3} \cdot \mathrm{H}_{2} \mathrm{O}$ 条件下同样 没有肽出现，但在 $80 \% \mathrm{ACN} / 3 \% \mathrm{NH}_{3} \cdot \mathrm{H}_{2} \mathrm{O}$ 条件下糖肽和 非糖肽全都出现. 我们考虑是因为糖肽与非糖肽在氧化 铝上的配基交换作用相似，所以在同一浓度氨水条件下 一起被洗脱. 通过这个实验表明配基交换作用对多肽有 保留作用。

\section{3 氧化铝富集糖肽方法的建立}

由于糖肽与其相对应非糖肽的根本区别在于两者 极性不同，所以根据上述得到的结论，我们在洗脱液中 加入适当浓度的氨水用以减弱氧化铝对多肽的配基交 换作用，再通过亲水洗脱模式，乙腈浓度由高到低分别 

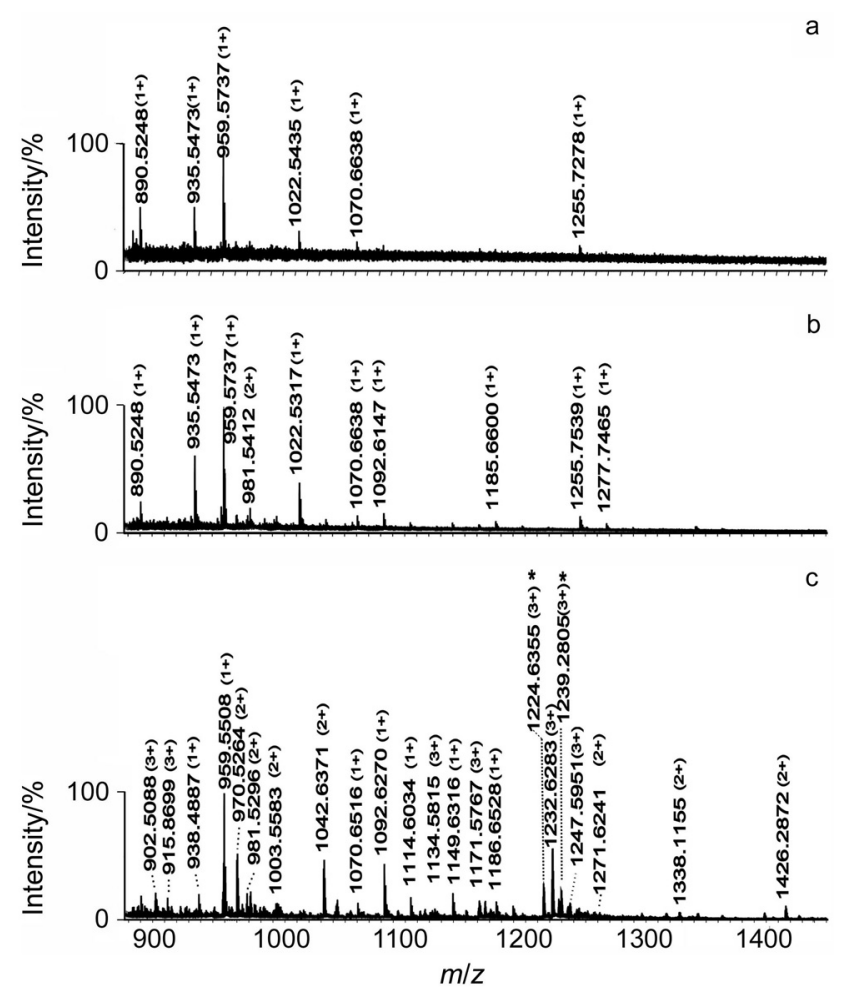

图 $2 \mathrm{HRP}$ 酶解液上样到氧化铝微柱后, 经(a) $80 \% \mathrm{ACN} / 1 \% \mathrm{NH}_{3} \cdot \mathrm{H}_{2} \mathrm{O}$, (b) $80 \% \mathrm{ACN} / 2 \% \mathrm{NH}_{3} \cdot \mathrm{H}_{2} \mathrm{O}$ 和(c) $80 \% \mathrm{ACN} / 3 \% \mathrm{NH}_{3} \cdot \mathrm{H}_{2} \mathrm{O}$ 洗脱后的质谱 图. “*” 为糖肽, 非糖肽以质荷比 $(m / z)$ 表示

Figure 2 Mass spectra of tryptic digest of HRP loaded into $\mathrm{Al}_{2} \mathrm{O}_{3}$ and subsequently eluted with (a) $80 \% \mathrm{ACN} / 1 \% \mathrm{NH}_{3} \cdot \mathrm{H}_{2} \mathrm{O}$, (b) $80 \% \mathrm{ACN} /$ $2 \% \mathrm{NH}_{3} \cdot \mathrm{H}_{2} \mathrm{O}$, and (c) $80 \% \mathrm{ACN} / 3 \% \mathrm{NH}_{3} \cdot \mathrm{H}_{2} \mathrm{O}$. Glycopeptides are marked with "*”. Other peptides are marked with their $m / z$ values

洗脱非糖肽和糖肽. 最终条件优化为: 取 $20 \mu \mathrm{L}$ HRP 酶 解液和 $10 \mu \mathrm{L} \mathrm{IgG}$ 酶解液旋干后分别溶解于 $70 \% \mathrm{ACN} /$ $1 \% \mathrm{NH}_{3} \cdot \mathrm{H}_{2} \mathrm{O}$ 中, 称取 $2 \mathrm{mg}$ 氧化铝, 装柱然后上样. 用 上样液淋洗 $45 \mu \mathrm{L}$ 后, $20 \mu \mathrm{L} 10 \% \mathrm{ACN} / 1 \% \mathrm{NH}_{3} \cdot \mathrm{H}_{2} \mathrm{O}$ 洗脱 收集, 用甲酸酸化后脱盐 ${ }^{[11]}$, 再进行质谱检测. 我们首 先考察了氧化铝对免疫球蛋白 $\mathrm{G}(\mathrm{IgG})$ 酶解液中糖肽的 富集效果. $\mathrm{IgG}$ 酶解液富集前直接检测的质谱图、经过氧 化铝富集后的质谱图和经商品化材料 Sepharose 富集后 的质谱图相比较如图 3 所示. 富集前在图 3a中只能找到 2 条糖肽, 经氧化铝富集后可以发现 12 条糖肽, 而经 Sepharose 富集后发现 4 条糖肽. 对 $\mathrm{IgG}$ 富集后发现, 不 仅检测到糖肽的数目明显增加, 而且与商品化材料 Sepharose 相比, 选用氧化铝作为富集材料还可以去掉 1344.2917 (5+), $1355.0881(5+)$ 和 $1359.2829(5+)$ 这样 大分子的非糖肽. 值得一提的是, 经氧化铝富集后糖肽 的检测信号也明显增加，如 $1215.9238(2+), 1317.9764$ $(2+), 1382.9946(2+)$ 和 $1480.0139(2+)$ 这些糖肽富 集后比富集前信号强度高 3.1 倍, 3.2 倍, 4.5 倍和 3.3 倍, 所有检测到的糖肽平均信号强度富集后要比富集前高 3.0 倍(表 1).

采用相同的方法处理, 以 HRP 酶解液为样品, HRP 的酶解液经过 $\mathrm{C} 18$ 去盐后从质谱中可以找到 7 条糖肽,
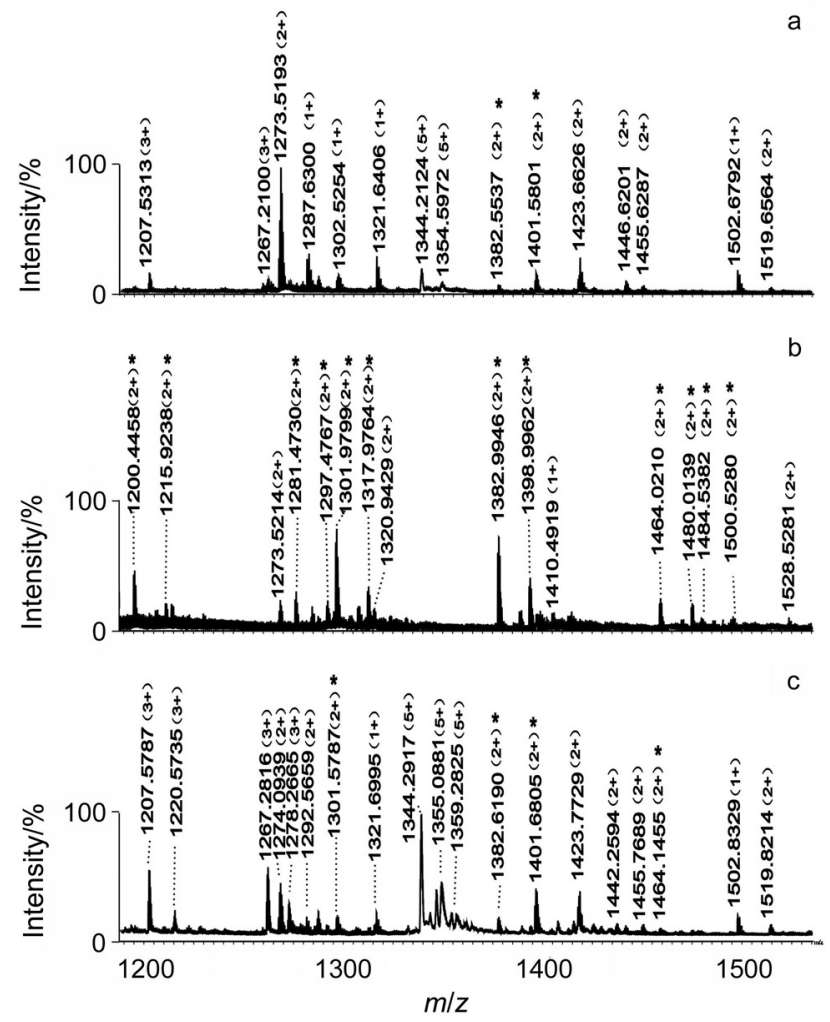

图 $3 \mathrm{IgG}$ 酶解液被三种材料处理后质谱图. (a) $\mathrm{C} 18$ 去盐后质谱图、(b) 氧化铝富集后质谱图、(c) Sepharose 富集后质谱图。“*”为糖肽, 非 糖肽以质荷比 $(\mathrm{m} / \mathrm{z})$ 表示

Figure 3 Glycopeptides from tryptic digest of $\operatorname{IgG}$ were enriched by different materials. (a) Glycopeptides enriched by $\mathrm{C} 18$; (b) glycopeptides enriched by $\mathrm{Al}_{2} \mathrm{O}_{3}$; (c) glycopeptides enriched by Sepharose. Glycopeptides are marked with “*”, other peptides are marked with their $\mathrm{m} / \mathrm{z}$ values

经氧化铝富集后可以发现 16 条糖肽. 与此相比, 经商品 化 Sepharose 材料富集后在质谱图中找到 14 条糖肽. 不 仅如此，我们根据表 1 还发现质谱检测到的糖肽信号同 样明显增高. 如 $1225.1486(3+), 1299.1204(2+)$ 和 $1408.5132(3+)$ 这些糖肽富集后检测到的信号强度要比 富集前高 3.8, 2.5 和 2.6 倍, 而所有检测到的糖肽的平均 信号强度富集后比富集前高 2.3 倍.

\section{4 结论}

我们发展了一种 $\mathrm{Al}_{2} \mathrm{O}_{3}$ 富集糖肽的新方法. 明确了 亲水作用和配基交换作用对多肽的保留影响. 根据上述 结论，我们优化并建立了氧化铝富集糖肽的方法. 在氧 化铝富集糖肽的实验中我们从 HRP 中共富集到糖肽 16 条, 而富集前只检测到 7 条糖肽, 富集后得到糖肽的数 量是富集前的 2 倍, 糖肽信号强度平均提高 2.3 倍, 而经 Sepharose 富集到 14 条糖肽. 从 $\operatorname{IgG}$ 中富集到 12 条糖肽, 而富集前只检测到 2 条, 富集后得到糖肽的数量是富集 前的 6 倍, 糖肽信号强度平均提高 3.0 倍, 而经 Sepharose 富集到 4 条糖肽. 与样品富集前和经商品化材 料富集后的糖肽数量相比较, 氧化铝在糖肽选择性和覆 盖率方面均有明显的优势. 所以通过实验证明氧化铝富 
集糖肽具有较好的选择性和覆盖率.

表 $1 \mathrm{IgG}$ 和 HRP 糖肽氨基酸序列, 质荷比, 富集后与富集前同一糖 肽质谱峰强度倍数关系

Table 1 Sequences and mass/charge ratio of glycopeptides from IgG and HRP and comparison of signal intensities between glycopeptides before and after enrichment

\begin{tabular}{|c|c|c|}
\hline IgG 糖肽氨基酸序列 & 质荷比 & $\begin{array}{c}\text { 同一糖肽质谱 } \\
\text { 峰强度倍数关系 }\end{array}$ \\
\hline EEQFNSTFR & $1199.7662(z=2)$ & 2.7 \\
\hline EEQYNSTYR & $1215.9238(z=2)$ & 3.1 \\
\hline EEQFNSTFR & $1281.4730(z=2)$ & 2.0 \\
\hline EEQFNSTFR & $1301.9799(z=2)$ & 2.2 \\
\hline EEQYNSTYR & $1317.9764(z=2)$ & 3.2 \\
\hline EEQFNSTFR & $1382.9946(z=2)$ & 4.5 \\
\hline EEQYNSTYR & $1398.9962(z=2)$ & 5.2 \\
\hline EEQFNSTFR & $1464.0210(z=2)$ & 3.7 \\
\hline EEQYNSTYR & $1480.0139(z=2)$ & 3.3 \\
\hline EEQFNSTFR & $1484.5382(z=2)$ & 2.3 \\
\hline EEQYNSTYR & $1500.5280(z=2)$ & 3.7 \\
\hline $\mathrm{HRP}$ 糖肽氨基酸序列 & 质荷比 & $\begin{array}{c}\text { 同一糖肽质谱 } \\
\text { 峰强度倍数关系 }\end{array}$ \\
\hline NVGL $\underline{N R}$ & $921.8337(z=2)$ & 3.0 \\
\hline $\begin{array}{l}\text { GLCPLNGㅁLSALVD } \\
\text { FDLR }\end{array}$ & $1202.7838(z=3)$ & 0.3 \\
\hline $\begin{array}{l}\text { GLIQSDQELFSSP } \\
\underline{N} \text { ATDTIPLVR }\end{array}$ & $1225.1486(z=3)$ & 3.8 \\
\hline $\begin{array}{l}\text { LHFHDCFVNGCDASI } \\
\text { LLD } \underline{N} T \text { TSFR }\end{array}$ & $1299.1204(z=2)$ & 2.5 \\
\hline $\begin{array}{l}\text { LHFHDCFVNGCDASI } \\
\text { LLD } \underline{N} T \text { TSFR }\end{array}$ & $1353.0941(z=3)$ & 1.3 \\
\hline $\begin{array}{l}\text { QLTPTFYD } \underline{N S C P N V S} \\
\text { NIVR }\end{array}$ & $1408.5132(z=3)$ & 2.6 \\
\hline $\begin{array}{l}\text { LY } \underline{N} F S N T G L P D P T L \underline{N} \\
\text { TTYLQTLR }\end{array}$ & $1662.2725(z=3)$ & 2.7 \\
\hline
\end{tabular}

\section{References}

[1] Ohtsubo, K.; Marth, J. D. Cell 2006, 126, 855.

[2] Fournier, T.; Porquet, D. Biochim. Biophys. Acta, Protein Struct. Mol. Enzymol. 2000, 1482, 157.

[3] Wang, Y. H.; Wu, S. L.; Hancock, W. S. Glycobiology 2006, 16, 514.

[4] Nihlen, U.; Montnemery, P.; Lindholm, H.; Lofdahl, C. G. Scand. J. Clin. Lab. Invest. 2001, 61, 341.

[5] Niwa, T. Mass Spectrom. Rev. 2006, 25, 713.

[6] Budnik, B. A.; Lee, R. S. Biochim. Biophys. Acta, Proteins Proteomics 2006, 1764, 1870 .

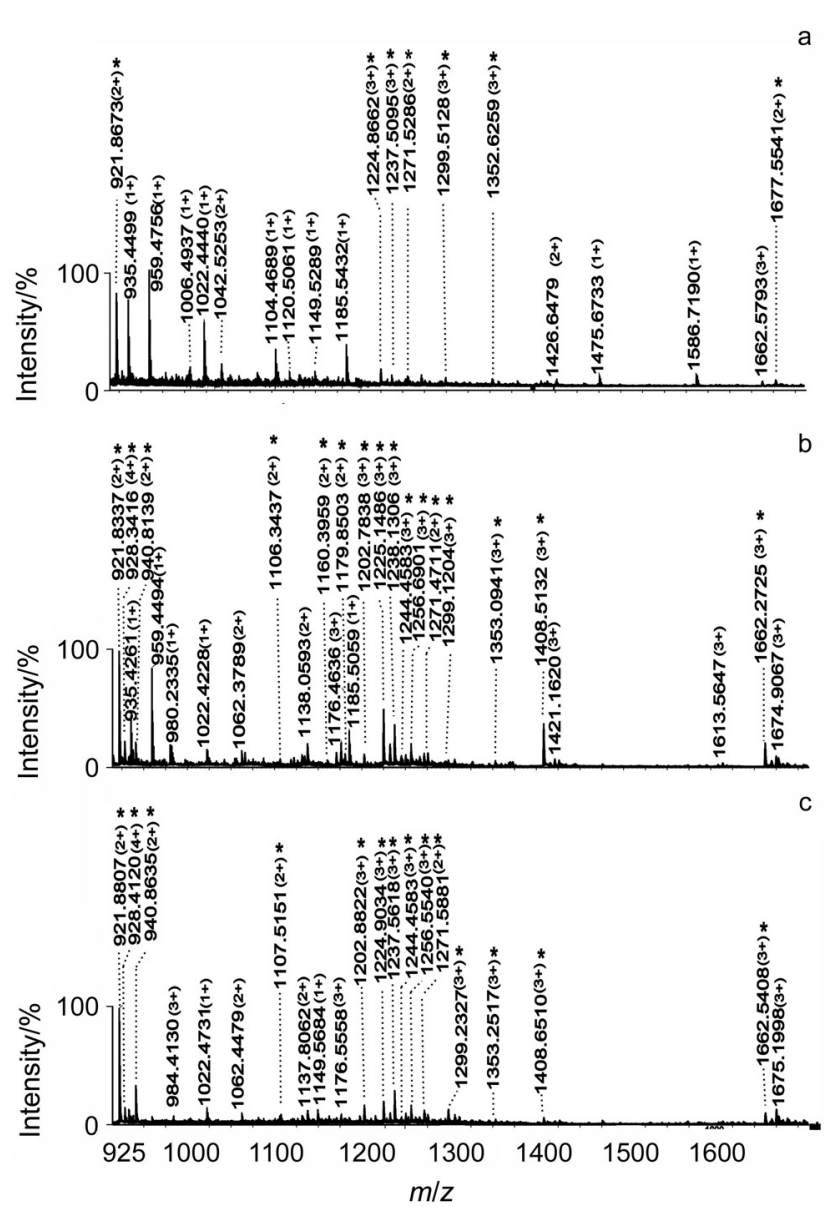

图 4 HRP 酶解液被三种材料处理后质谱图. (a) C18 去盐后质谱图、 (b)氧化铝富集后质谱图、(c) Sepharose 富集后质谱图. “*”为糖肽, 非 糖肽以质荷比 $(\mathrm{m} / \mathrm{z})$ 表示

Figure 4 Glycopeptides from tryptic digest of HRP were enriched by different materials. (a) Glycopeptides enriched by $\mathrm{C} 18$; (b) glycopeptides enriched by $\mathrm{Al}_{2} \mathrm{O}_{3}$; (c) glycopeptides enriched by Sepharose. Glycopeptides are marked with "*”, other peptides are marked with their $m / z$ values

[7] Witze, E. S.; Old, W. M.; Resing, K. A.; Ahn, N. G. Nat. Methods 2007, 4, 798

[8] Zhang, Q. B.; Tang, N.; Brock, J. W. C.; Ames, J. M. J. Proteome Res. 2007, 6, 2323.

[9] Hirabayashi, J. Glycoconjugate J. 2004, 21, 35.

[10] Grun, M.; Kurganov, A. A.; Schacht, S. J. Chromatogr. A 1996, $740(1), 1$.

[11] Yan, J.; Li, X.; Yu, L.; Jin, Y.; Zhang, X.; Xue, X.; Ke, Y.; Liang, X. Chem. Commun. 2010, 46, 5488.

[12] Wan, H.; Yan, J.; Yu, L.; Sheng, Q. Y.; Li, X. L.; Liang, X. M. Analyst 2011, 136, 4422 . 